\title{
Impact of 3D magnetic field structure on boundary and divertor plasmas in stellarator/heliotron devices
}

M. Kobayashi ${ }^{1}$, Y. Feng ${ }^{2}$, Y. Xu ${ }^{3}$, F.L. Tabares ${ }^{4}$, K. Ida ${ }^{1}$, O. Schmitz ${ }^{5}$, T.E. Evans ${ }^{6}$, H. Frerichs ${ }^{5}$, Y. Liang ${ }^{7}$, A. Bader ${ }^{5}$, K. Itoh ${ }^{1}$, H. Yamada ${ }^{1}$, Ph. Ghendrih ${ }^{8}$, G. Ciraolo ${ }^{8}$, D. Tafalla ${ }^{4}$, A. Lopez-Fraguas ${ }^{4}$, H.Y. Guo ${ }^{6,9}$, Z.Y. Cui ${ }^{3}$, D. Reiter ${ }^{7}$, N. Asakura ${ }^{10}$, U. Wenzel ${ }^{2}$, S. Morita ${ }^{1}$, N. Ohno ${ }^{11}$, B.J. Peterson ${ }^{1}$, and S. Masuzaki ${ }^{1}$

${ }^{1}$ National Institute for Fusion Science,322-6 Oroshi-cho, Toki 509-5292, Japan

${ }^{2}$ Max-Planck-Institute fuer Plasmaphysik, D-17491 Greifswald, Germany

${ }^{3}$ Southwestern Institute of Physics, P. O. Box 432, Chengdu 610041, China

${ }^{4}$ Laboratorio Nacional de Fusion. Ciemat, Madrid, Spain

${ }^{5}$ University of Wisconsin-Madison, WI, USA

${ }^{6}$ General Atomics, P.O. Box 85608, San Diego, California 92186-5608, USA

${ }^{7}$ Forschungszentrum Jülich GmbH Institut für Energie- und Klimaforschung - Plasmaphysik, Jülich, Germany

${ }^{8}$ IRFM, CEA Cadarache ST Paul Lez Durance, France

${ }^{9}$ Institute of Plasma Physics, CAS, Hefei, China

10 Japan Atomic Energy Agency, Rokkasho, Aomori 039-3212, Japan

${ }^{11}$ Nagoya University, Nagoya 464-8603, Japan

$\underline{\text { PACS}}$; 52.55.Hc, 52.55.Fa, 52.55.Rk, 52.25.Fi

PSI-21 keywords; Divertor plasma, Stochastic boundary, Magnetic topology, Island divertor, $3 \mathrm{D}$ effect

\section{Abstract}

This paper overviews recent progress on the experimental identification and physics interpretation of 3D effects of magnetic field geometry on divertor transport. The 3D effects are elucidated as a consequence of competition between transports parallel (//) and perpendicular $(\perp)$ to magnetic field, in open field lines cut by divertor plates, or in magnetic islands. The competition has strong impacts on divertor functions, such as determination of density regime, impurity screening, and detachment control. The effects of magnetic perturbation on the edge electric field and turbulent transport are also discussed. Based on the experiments and numerical simulations, key parameters governing the 3D transport physics for the individual divertor functions, e.g. pumping efficiency through divertor density regime, impurity screening and detachment control, are discussed. 


\section{Introduction}

The divertor optimization with the 3D edge magnetic field structure is inevitable in helical devices due to intrinsic non-axisymmetric magnetic configurations $[1,2,3,4,5,6]$, and also in tokamaks with the application of symmetry breaking magnetic perturbation (MP) fields aimed at edge transport control $[7,8,9]$ or at ELM mitigation or suppression $[10,11,12$, $13,14,15,16,17,18]$. Understanding of the $3 \mathrm{D}$ effects is, therefore, prerequisite to divertor optimization for a future reactor, exploring advanced magnetic configurations. Recent development of 3D numerical simulation codes, EMC3-EIRENE [19,20], TOKAM-3X, -3D [21,22,23], E3D [24], FINDIF [25] etc., as well as systematic experiments [26,27,28,29,8] have revealed various transport aspects in the 3D magnetic configurations. In these contexts, this paper overviews the recent progress on the experimental identification and physics interpretation of 3D effects of magnetic field geometry on divertor transport, and suggests key parameters that control the effects, in order to provide a perspective on divertor optimization for future reactors.

The 3D effects have been intensely investigated so far in various devices and these investigations have made significant progress in the understanding of transport features $[7,8,28,30,31,32,33,34,35]$. The modifications of transport are caused by the stochasticity of field lines or by the 3D deformation of magnetic flux tubes [36,37]. The outermost region is called the laminar zone [38] or edge surface layers [1], where parallel correlation along field lines still persists to some extent, i.e., $L_{K}$ (Kolmogorov length) $>L_{C}$ (connection length to divertor plates). Particularly in the present study, in order to obtain a simple picture, the 3D effects are defined as follows. The 3D effects emerge when the //-transport starts to compete with the $\perp$-transport to deliver particle, momentum or energy from upstream (i.e., around the last closed flux surface, LCFS, or around midplane in X-point divertor) to the downstream (i.e., divertor region). This situation occurs either in open field lines or in magnetic islands, as shown in Fig.1. The //-transport time becomes finite along an open field line or circulates inside the island, which then competes with the $\perp$ - transport. Here we consider the case,

$$
\Gamma_{/ /}\left(\frac{B_{r, \theta}}{B_{t}}\right)^{2} \sim \Gamma_{\perp},
$$

where $B_{r, \theta}, B_{t}$ are the radial or poloidal component of the magnetic field and toroidal field, 
respectively. $\Gamma_{/ /, \perp}$ represents the flux of any physical quantity (particle, momentum, energy) that is parallel or perpendicular to magnetic field lines, respectively. The ratio $\Gamma_{/ /} / \Gamma_{\perp}$ ranges from $10^{5}$ to $10^{8}$, depending on the physical quantities as well as on the plasma parameters. In the scrape-off layer (SOL) of 2D axi-symmetric X-point divertor tokamaks, (in that case we take $B_{\theta}$ in eq.(1) instead of $B_{r}$ ), where typically $\frac{B_{\theta}}{B_{t}} \sim 0.1$. In this case, we have the relation, $\Gamma_{/ /}\left(\frac{B_{\theta}}{B_{t}}\right)^{2}>>\Gamma_{\perp}$, thus the //-transport dominates over $\perp$ transport. On the other hand, in the stochastic layer in helical devices, in tokamaks with a resonant magnetic perturbation (RMP) field, or in the island divertor configuration, where typically $\frac{B_{r}}{B_{t}}=10^{-4} \sim 10^{-3}$, the situation allows the $\perp$-transport to contribute substantially to deliver the plasma quantity of particles, momentum and energy, and thus $\Gamma_{/ /}\left(\frac{B_{r}}{B_{t}}\right)^{2} \sim \Gamma_{\perp}$. The parameter domain in which this effect becomes significant is elucidated in Fig.8 in ref.[39].

For momentum transport, which is a vector field, the direction of the flow or of the field line connection is also important. When the flow fields that have (toroidally) opposite streaming directions and thus interact with each other due to the proximity of the counter connecting field lines, they loses the //-momentum via dissipation caused by $\perp$-viscosity. Such a situation can occur in the island divertor [39] or in the stochastic layer [32], as shown in Fig.2. In the 2D axi-symmetric X-point divertor, the separation of counter directional flux tubes, i.e., inner and outer divertor legs, are usually large, and thus the momentum loss effect is considered to be negligible.

The enhancement of $\perp$-transport also occurs in the situation where short and long connection length flux tubes are touching each other and they are "squeezed" due to the magnetic shear, as shown in Fig.3. In this case, the exchange of physical quantities such as particle and energy between the different flux tubes is considered to be enhanced when there is substantial density due to the enlarged interface between them.

It is noted that, in closed nested flux surfaces the //-transport uniformly distributes plasma parameters along the flux surfaces. In such cases, the //-transport does not contribute 
to "net" transport from the LCFS to divertor plates (or "net" radial direction), even with non-axisymmetric deformation of the magnetic flux surfaces.

In the following sections, we investigate how these 3D effects are reflected in the experimental observations and then give rise to possible impacts on divertor functions.

\section{Impact on divertor density regime}

The divertor density regime in the $2 \mathrm{D}$ axi-symmetric $\mathrm{X}$-point divertor configuration is known for strong recycling enhancement at the divertor region, which is sustained with the sufficient upstream plasma pressure and energy input needed for the ionization process and with effective neutral confinement [40]. The divertor (downstream) density and temperature are strongly coupled to the upstream (LCFS or midplane) density, for example, $n_{\text {down }} \propto n_{\text {up }}{ }^{3}, T_{\text {down }} \propto n_{u p}{ }^{-2}$, which is derived from the pressure conservation along flux tubes between the upstream and the downstream. Such a density regime is called the high-recycling regime or conduction limited regime and the relation has been confirmed in experiments [41, 42, 43, 44, 45, 46, 47]. However, in the 3D divertor configurations such as helical devices, W7-AS [48,49], LHD [50, 51] and tokamaks with an RMP, TEXTOR-DED [52], it has been sometimes found that the dependency becomes modest, i.e., $n_{\text {down }} \propto n_{\text {up }}{ }^{\sim}, T_{\text {down }} \propto n_{\text {up }}{ }^{-\sim 1}$, and also that the downstream density never exceeds the upstream density, $n_{\text {down }}<n_{u p}$, while in the $2 \mathrm{D}$ configuration usually we observe $n_{\text {down }}>>n_{u p}$. In the analysis in this section we restrict ourselves to the conduction limited regime when we refer to the high recycling regime, and also assume that most of the ionization takes place at the divertor plate, i.e. the plasma is attaches to the divertor plate. The upstream densities in LHD, W7-AS and TEXTOR-DED are taken at the LCFS, where the poloidal variation of the density is usually small. Such a modest dependency is observed in the condition with SOL collisionality, $v_{S O L}{ }^{*}=L_{C} / \lambda_{e e}$, more than 10 , where one expects the high recycling regime in tokamaks, and with the divertor plasma temperature, $T_{d i v}$, more than $10 \mathrm{eV}$, where momentum removal via $\mathrm{CX}$ is considered low. Here $L_{C}$ and $\lambda_{e e}$ are field line connection length and electron mean-free path, respectively. It is also noted that in the devices 
mentioned above, $T_{\text {down }} \geq 10 \mathrm{eV}$ before the detachment transition, while significant momentum loss via plasma neutral interaction is expected at $T_{\text {down }} \leq 5 \quad[53,54,55]$. Therefore, the effect of neutrals on the momentum loss is considered to be small in the present case.

The phenomena have been interpreted as due to the loss of //-momentum as shown in Fig.2, which was first investigated in the island divertor configuration of W7-AS [49], using the 3D edge transport code EMC3-EIRENE [56,20]. In the W7-AS island divertor, due to the smallness of the island, the counter-streaming plasma flows along the island fans interact with each other and lose //-momentum, as discussed in the Section 1. The effect is elucidated in Fig. 4 by including $\perp$-loss term in the momentum equation along flux tubes. One sees that with an increasing momentum loss the upstream and downstream coupling becomes weak and then the density dependence changes from $n_{\text {down }} \propto n_{u p}{ }^{3}, T_{\text {down }} \propto n_{u p}{ }^{-2}$ to $n_{\text {down }} \propto n_{u p}^{\sim 1}, T_{\text {down }} \propto n_{u p}^{-\sim 1}$. An enhanced loss of //-momentum has also been identified in the stochastic layer of LHD, where the radial interaction between counter-streaming flows is caused both by the compression of flux tubes near helical coils and the "squeezing" effect of counter-directional flux tubes (i.e., flows) due to the strong magnetic shear $[32,57,58]$. Numerical analysis in HSX has also shown the absence of a high recycling regime due to the momentum loss in the edge island structure [59].

The effect of //-momentum loss can be formulated as the ratio between the momentum transport time parallel and perpendicular to the magnetic field,

$$
\frac{\tau_{m / /}}{\tau_{m \perp}}=\frac{D_{\perp} L_{/ /}}{V_{/ /} \lambda_{m}{ }^{2}},
$$

where $D_{\perp}, L_{/ /}, V_{/ /}$are the perpendicular particle diffusivity, //-characteristic scale length for momentum transport, i.e., connection length $\left(L_{C}\right)$ or Kolmogorov length $\left(L_{K}\right)$ of field lines and parallel plasma velocity, respectively. $\lambda_{m}$ is the $\perp$-characteristic scale length for momentum transport, i.e., the distance between the counter-streaming flows. The ratio corresponds to the momentum loss factor $f_{m}$ discussed in refs.[60,32]. The larger the ratio is, the larger the $\perp$-loss of //-momentum is. 
In TEXTOR-DED with the $m / n=6 / 2$ mode of RMP operation, the detailed analysis of the 3D edge transport simulation has shown that the replacement of //-energy flux $\left(q_{/ / e}\right)$ with $\perp$-transport $\left(q_{\perp e}\right)$, as discussed in Fig.1, as well as with the convection flux $\left(q_{/ / e, \text { conv }}\right)$ due to the substantial upstream ionization source, are responsible for the modification of this density regime [61]. The effect of convection energy flux was also pointed out in ref.[62]. Both effects lead to the reduction of //-conduction energy $(q / / e$, cond $)$ and weaken the upstream and downstream coupling as elucidated in ref.[63], such that $n_{\text {down }} \propto f_{\text {cond }}^{6 / 7}$, where $f_{\text {cond }}$ represents a fraction of the conduction component out of all parallel energy flux. The contribution of $q_{\perp e}$ can be formulated as,

$$
\frac{q_{\perp e}}{q_{/ / e}}=\frac{n \chi_{\perp e}}{\left(B_{r} / B_{t}\right)^{2} \kappa_{0 e} T_{e}^{2.5}},
$$

where $\chi_{\perp e}, \kappa_{0 e}$ are the perpendicular heat diffusivity and the coefficient for //-heat conductivity, respectively. The effect of convection energy flux, $q_{/ / \text {e, conv }} / q_{/ /}$, cond is strongly dependent on the detailed distribution of plasma parameters and the divertor geometry, which affect the neutral penetration into the edge plasma as discussed in details in $[64,65,66,67]$. In some 3D divertor configurations, such as TEXTOR-DED, which have an "open" divertor configuration, the neutral penetration into the upstream region approaches $\sim 50 \%$ of the total recycling flux $[52,61]$. This upstream source gives rise to a substantial convection flux, $q_{/ / e, \text { conv }} / q_{/ / e, \text { cond }} \sim 1$ [33]. The estimation of $q_{/ / e, \text { conv }} / q_{/ / e, \text { cond }}$ is, however, not straightforward and one needs detailed numerical analysis taking into account the 3D geometry of edge plasma and of divertor and baffle structures. The larger the $q_{\perp e} / q_{/ / e}$ or $q_{/ / e, c o n v} / q_{/ / e, \text { cond }}$, the weaker the density dependence of the divertor plasma parameters, approaching $n_{\text {down }} \propto n_{u p}^{\sim 1}, T_{\text {down }} \propto n_{u p}^{-\sim 1}$.

The strong upstream and downstream coupling, i.e., the high recycling regime, is recovered, if either or both of $\tau_{m / /} / \tau_{m \perp}$ and $q_{\perp e} / q_{/ / e}$ are considered to be small enough to maintain the robust upstream and downstream coupling. This has been achieved in Tore Supra [68], TEXTOR-DED with an $\mathrm{m} / \mathrm{n}=3 / 1$ mode [69]. The numerical simulations with 
EMC3-EIRENE on W7-X and on HSX with a large island size have also shown a clear high recycling regime [31,59].

In the present analysis, we use the divertor density obtained from probe measurements as an indication of whether the density dependence is the high-recycling regime or not, instead of divertor particle flux, which might be a more robust quantity [43]. We have confirmed that the same feature is observed also in the particle flux measurements in the experiments and also that the divertor plasma temperature is relatively high, $\gtrsim 10 \mathrm{eV}$ before the detachment transition for all the data referred to in the present analysis. Therefore, the estimation of the divertor density is considered to be reliable.

Figure 5 summarizes the density regime of various devices in terms of $\tau_{m / /} / \tau_{m \perp}$ and $q_{\perp e} / q_{/ / e}$, where the results from numerical simulation are plotted with dashed lines. The $\lambda_{m}$ has been estimated as $\lambda_{m} \approx 2 \pi a / m$, assuming that the counter streaming flows are regulated in the poloidal direction as shown in Fig.2. The situation is different in LHD. In LHD the strong magnetic shear squeezes the flux tubes and thus $\lambda_{m}$ becomes much smaller e.g. a few cm [32], as depicted in Fig.3. For the calculations of $\tau_{m / /} / \tau_{m \perp}$ and $q_{\perp e} / q_{/ / e}$, the half values of the plasma parameters at the LCFS, i.e., $0.5 \times T_{L C F S}$ and $0.5 \times n_{L C F S}$ are assumed as representative values. It is certainly much better to analyze the 3D structure of each plasma parameter in the SOL to estimate the $\tau_{m / /} / \tau_{m \perp}$ and $q_{\perp e} / q_{/ / e}$ in the 3D divertor configuration. But it is almost impossible to obtain such profiles in all devices. Instead, we have taken the half values of the parameters at the LCFS as representative values. Since the precise estimation of $\tau_{m / /} / \tau_{m \perp}$ and $q_{\perp e} / q_{/ / e}$ needs to be done by integration along the 3D structure of the SOL from the upstream to the downstream, neither the values at the LCFS nor at the divertor target are very appropriate for the calculation. Instead, here we assume that the usage of an intermediate value might be as reasonable as possible for the estimation and it is the best that we can do at present. While we are aware that there is a limitation of this method for accurate estimation of the SOL characteristics, the simplification enables us to obtain an overview of the global trend of SOL feature of each device. Certainly, 
it is still left for future works to estimate precisely the values of $\tau_{m / /} / \tau_{m \perp}$ and $q_{\perp e} / q_{/ / e}$ with 3D integrations. The present results should be considered as very rough estimation and just used to see the rough tendency of the SOL characteristics of each device. It has been also assumed that for the devices with a stochastic edge layer $L_{/ /} \approx L_{K} \sim \pi R q$ based on the argument in ref.[38], while for the island divertor configurations (W7-AS/X) $L_{/ /} \approx L_{C} \sim 100$ $\mathrm{m}[48,70]$. A Mach number of 0.5 is used for the calculation of $V_{/ /}$. The estimations of $\tau_{m / /} / \tau_{m \perp}$ and $q_{\perp e} / q_{/ / e}$ are rather rough, but Fig.5 shows a clear tendency of the transition from cases capable of obtaining the high recycling regime to cases with absence of a high recycling regime as $\tau_{m / /} / \tau_{m \perp}$ and $q_{\perp e} / q_{/ / e}$ are increased. It is noted that for the case of ITER, it has been shown that the strong magnetic shear deforms the flux tubes significantly and reduces the $\lambda_{m}$ down to $\sim$ several $\mathrm{cm}$ [11]. If we take the small value for $\lambda_{m}$, then $\tau_{m / /} / \tau_{m \perp}$ for the ITER case with an RMP will increase and become closer to the border between the cases with and without high recycling regimes. However, there is a fundamental difference between the X-point poloidal divertor with an RMP and the other devices, LHD, W7-AS/X, Tore Supra, TEXTOR-DED, etc. In the former case, the dominant diverting field is still $B_{\theta}$, since $B_{\theta} / B_{t} \sim 0.1$ and $B_{r} / B_{t} \sim 10^{-3}$. In the latter case, the radial projection of //-flux due to $B_{r}$ directly competes with the $\perp$-transport. As long as considering //-flow, even in the case with an RMP the flow direction might still be predominantly determined by $B_{\theta} / B_{t}$ with a small modulation due to $B_{r} / B_{t}$, and it might not cause significant effects on the //-momentum transport. However, the flow field formation is a complex process depending on the ionization distribution as well as on drift as discussed in ref.[64]. In addition, there have been discussions on the energy and particle loss into the private flux region (PRF) in the 2D poloidal divertors, which affects the particle and energy deposition profiles on the divertor plates $[64,71,72,73,74]$, and recently it is even found to be enhanced [75], while fewer discussions are made on the possible effects on momentum transport. We need further detailed investigation both in experiments and numerical simulations for these issues. 


\section{Impact on SOL \& divertor impurity transport}

There have been many observations of impurity screening or core decontamination in tokamaks with RMP application [76,77,78], and in helical devices with a stochastic layer $[79,80]$ or with the island divertor configurations $[81,82]$. It is not clear yet at all whether these phenomena are caused by the edge impurity screening or attributed to core transport or some other effects such as a change of source characteristic, etc. Here we discuss the 3D geometrical effects on the edge impurity transport. A simple picture of impurity screening in the 3D divertor configuration may be drawn as follows: Based on the //-momentum balance equation for the impurity, the resultant profile of the impurity density is considered to be determined by a competition between the friction force and the ion thermal force acting on the impurity [63]. That is,

$m_{z} \frac{\partial V_{z / l}}{\partial t} \approx m_{z} \frac{V_{/ /}-V_{z / l}}{\tau_{s}}+C_{i} \nabla_{/ /} T_{i}+\ldots$

where $m_{z}, V_{z / /}, V_{/ /}, \tau_{s}$ are the mass of the impurity, the //-velocity of the impurity, the //-velocity of background plasma, and the collision time (slowing down time of the impurity) between the impurity and the plasma, respectively. The friction force drags the impurity towards the plasma flow direction (i.e., assuming that the flow is directed towards the divertor plates) through the friction between the plasma and the impurity. On the other hand, the ion thermal force drives the impurity upstream because the //-temperature gradient points upstream and toward higher temperatures. With $B_{r}$ from MP, the outward (radial) plasma flow can be enhanced due to the radial projection of $V_{/ /}$. This also leads to an enhancement of recycling due to a reduced particle confinement time, and thus results in a colder and denser edge region, which reduces the collision time between the plasma and the impurity, $\tau_{s}$. Since the friction term has the dependence of $\sim \frac{V_{\| /}-V_{z / l}}{\tau_{s}}$, the above effects lead to an enhancement of the friction force. It is also noted that effective screening is often observed in the higher density range, i.e., at higher collisionality $[83,79,80,84$,$] . This is also consistent$ with the picture of the friction force that is inversely proportional collision time $\tau_{s}$. The 
effects of the friction force have been pointed out so far in many publications $[85,86,87,88,89,90]$, and here we use the following expression to measure the effect,

$\frac{D_{s t}}{D_{\perp}}=\frac{\left(B_{r} / B_{t}\right)^{2} V_{/ /} L_{/ /}}{D_{\perp}}$,

where $D_{s t}$ represents the enhancement of the radial particle transport via a projection of the //-plasma flow, and $D_{\perp}$ is $\perp$-particle diffusivity, which ranges from 0.4 to $1.0 \mathrm{~m}^{2} / \mathrm{s}$, depending upon the device. Here, however, one should also note that a highly collisional plasma also develops a //-temperature gradient, and thus an ion thermal force. In the island structure that appears in the $3 \mathrm{D}$ divertor configurations, it has been pointed out that //-conductive ion energy flux $\left(\propto T_{i}^{2.5} \nabla_{/ /} T_{i}\right)$ can be reduced while increasing $\perp$-conductive energy flux $\left(\propto n \nabla_{\perp} T_{i}\right)$ under high density and low temperature condition. It has been shown that this effect becomes remarkable when $n \chi_{\perp}>\kappa_{i 0} T_{i}^{2.5}\left(B_{r} / B_{t}\right)^{2}$, and the increased $\perp$-conductive energy flux can provide a bypass through which the //-conductive energy flux can be reduced [60]. It thus relaxes the //-temperature gradient. This effect has been pointed out in the numerical simulation with EMC3-EIRENE on W7-AS [60] and also later confirmed in LHD [79]. The effect can be formulated as [60],

$$
\frac{q_{\perp i}}{q_{/ / i}}=\frac{n \chi_{\perp i}}{\left(B_{r} / B_{t}\right)^{2} \kappa_{0 i} T_{i}^{2.5}},
$$

similar to eq.(3) but now for ions. In the case of a 2D axi-symmetric X-point divertor, where $B_{\theta} / B_{t} \sim 0.1$ is used instead of $B_{r} / B_{t}$ as discussed in section $1, \frac{q_{\perp i}}{q_{/ / i}}<<1$ and the suppression of ion thermal force is not foreseen. In the 3D divertor, on the other hand, due to a very small $B_{r} / B_{t}=10^{-4} \sim 10^{-3}$, the condition $\frac{q_{\perp i}}{q_{/ / i}} \sim 1$ is fulfilled. The modelling analysis that takes into account these effects has been conducted in comparison with the carbon emission measurements in LHD [79], where qualitatively good agreement has been found for the CIV profile and density dependence of CIII, CIV, and CV. This indicates the existence of the screening mechanisms in the edge stochastic layer. 
The systematic experiments in LHD varying the radial thickness of the stochastic layer have shown that a thicker stochastic layer seems more effective for screening the impurity [91]. This is considered to be due to an increase of the radial extension of the friction force dominant region. Since the friction dominant region favours low temperature and high density condition with higher plasma velocity, the region is located downstream. On the other hand, the upstream tends to be a thermal force dominant region. The thicker stochastic layer can provide a thicker friction force region, which thus reduces the impurities that penetrate directly into the thermal force dominant region as neutrals that then build up upstream.

Figure 6 summarizes the observations of impurity screening in experiments or in numerical simulations such as for W7-X and HSX, in terms of $\frac{D_{S t}}{D_{\perp}}$ and $\frac{\lambda_{s t-S O L}}{\lambda_{i m p}}$, where $\lambda_{s t-S O L}$ and $\lambda_{\text {imp }}$ are the radial thickness of the stochastic layer or the island divertor SOL and the impurity penetration length. For $\lambda_{i m p}=V_{i m p}^{0} /\left(\langle\sigma v\rangle_{i o n} n\right)$, where $V_{i m p}^{0}$ and $\langle\sigma v\rangle_{i o n}$ are the velocity of the neutral impurity and a rate coefficient of neutral impurity ionization, respectively, we used $0.05 \mathrm{eV}$ for the neutral impurity injection energy and $0.25 T_{L C F S}, 0.25 n_{L C F S}$ as representative plasma parameters for calculations of the index. The selection of the factor 0.25 , instead of 0.5 as done in Section 2, is to take into account the fact that neutral impurities come from outside of the plasma and interact with plasma at more peripheral region. Due to the same arguments in Section 2, however, this should be considered as a very rough estimate to obtain an overview of the SOL characteristics between different machines, and more precise calculations using 3D plasma structure should be carried out in the future. In the present analysis, carbon is assumed as the impurity. It is noted that Fig.6 does not show a clear tendency in terms of the parameters introduced here. A similar plot but with a horizontal axis replaced by $\frac{q_{\perp i}}{q_{/ / i}}$ (not shown here) does not show any clear parameter dependence with respect to impurity screening, either. This means that, we still have to analyse further the definition of screening efficiency, e.g. the ratio of impurity influx at the LCFS to the impurity source at the PFC, and also to investigate the plasma parameter (such as density or collisionality) dependence of the efficiency. It is also noted that there exist 
hidden parameters that characterize the impurity transport, such as injection energy, recycling location, drift, electric field, turbulence transport, etc. Indeed, the clear changes of the electric field as well as the turbulent transport have been observed with RMP application [101] as discussed in Section 5 .

\section{Impact on detachment}

Because of the fast //-transport with respect to $\perp$-transport, it is considered that the change of the magnetic geometry can modify the edge radiation structure. This has been demonstrated in the Tore Supra ergodic divertor with an MP applied to lower hybrid current drive (LHCD) discharges [92]. It has been found that with relatively low density, 0.12 $\leq \rho_{m}=\pi a^{2}<n_{e 20}>/ I_{p} \leq 0.22$, in terms of the Greenwald density fraction, a MARFE is obtained during MP application + LHCD, where the radiated power is almost doubled compared to the phase without an MP. The MARFE is positionally stable at the high field side with radiation more than $90 \%$ of the total input power [92].

In W7-AS, it has been found that with proper choice of the edge island geometry, i.e., large island and with short connection length, an operation domain with steady state partial detachment is realized [93,94]. In such cases, the 3D numerical simulation with EMC3-EIRENE shows that the radiation region moves to the inboard side and is peaked around island X-points. In the case with a small island and a long connection length, otherwise, the intense radiation remains in front of the divertor plate. In this case the plasma stored energy significantly decreases after detachment transition and the radiated power strongly oscillates. Finally the discharge terminates due to a deep inward shift of the radiation front. We call the case unstable detachment in comparison to the steady state partial detachment mentioned above, which is referred to as stable detachment. The operation domain for stable detachment is shown in Fig.7, in terms of the distance between the divertor plate and the LCFS, $\triangle x_{L C F S-d i v}$, and $L_{C}$ [94]. The divertor radiation lowers the temperature at the recycling zone and the island becomes transparent to the neutral penetration into the core region. It is considered that the sudden increase of neutral penetration into the core at the detachment transition drives an instability resulting in the collapse, while in the case of the larger island with the inboard side radiation instead of the 
divertor radiation, the island has a screening effect against the neutral penetration and thus stabilizes detachment [94]. This is probably a similar argumentation to that made in axi-symmetric tokamak devices.

In LHD, it has been found that the application of an RMP with $\mathrm{m} / \mathrm{n}=1 / 1$, which creates a remnant island structure in the stochastic region, has a stabilizing effect on the detachment [95]. In the present analysis, the stable detachment means that the detached phase can be sustained with gas puff feedback control for more than several seconds until the end of the NBI heating with almost constant radiated power. Without an RMP, otherwise, the sudden increase of the radiation can not be stopped even if we turn off the gas puff, and the discharge ceases. We call this case unstable detachment. The divertor probe array measurements show that the particle flux profile has $n=1$ mode structure in the toroidal direction during detachment [96]. The 3D numerical simulation with EMC3-EIRENE shows that with RMP application intense radiation is formed along the trajectory of the X-point of the $\mathrm{m} / \mathrm{n}=1 / 1$ island, while without the RMP the radiation is localized at the inboard side throughout the torus [95]. The radiation distribution measurements with both AXUVD as well as imaging bolometer show the signature of the intense $\mathrm{X}$-point radiation, confirming, at least qualitatively, agreement with the code prediction for the modification of the $3 \mathrm{D}$ radiation structure $[96,97,98]$. The operation domain for the stable detachment with an RMP is shown in Fig.8 in terms of the distance between the island X-point and the LCFS, $\Delta x_{L C F S-i s l a n d}$, and the RMP strength, $B_{r} / B_{t}$ [95]. It is seen that in order to realize stable detachment one needs both the larger $\Delta x_{L C F S-i s l a n d}$ and $B_{r} / B_{t}$. Although the mechanism of the stabilization is under investigation, the similar tendency in $\Delta x_{L C F S-i s l a n d}$ as that in $\Delta x_{L C F S-d i v}$ indicates the importance of the decoupling between core and divertor recycling region to avoid instability caused by the sudden neutral penetration at the detachment transition. The stability analysis with a perturbation method also shows that flattening of the temperature profile at the island plays a key role to prevent inward penetration of radiation [99]. It is also found that the larger the $B_{r} / B_{t}$ is, the lower the detachment onset density, which is a similar trend observed in the MARFE onset in Tore Supra discussed above. 
In TEXTOR-DED, it has been observed that application of a rotating RMP to an NBI heated plasma shifts the MARFE onset density to a higher range compared to the case without an RMP [100]. The experimental results show that the threshold of the MARFE onset strongly depends on both the level and the poloidal distribution of recycling at the high field side, where the RMP coils are located. It is considered that both the smoothing of recycling and poloidal flow of plasma particles with moving field lines of a rotating RMP can increase the onset density of a MARFE by avoiding localized cooling of the plasma in front of the RMP coils [100].

The experimental observations shown above clearly indicate a possibility to control the edge radiation structure and the detachment stability in the 3D divertor configurations, with key controlling parameters such as the distance between the edge radiation region and the confinement region, $\Delta x_{L C F S-i s l a n d, d i v}$, the RMP amplitude, $B_{r} / B_{t}$, and the rotating frequency of the RMP, $f_{R M P}$.

\section{Impact on edge electric field and turbulence}

The change of the edge magnetic geometry by the application of an RMP can affect turbulence via several effects caused by the magnetic field braiding: the radial component of the field lines, $B_{r}$, can induce radial electron current, induce radial transport such as $D_{s t}$ (eq.(5)), modify the parallel wavelength of the modes, $k_{/ /}\left(\nabla_{/ /}=\left(\vec{B}_{0}+\vec{B}_{r}\right) \bullet \vec{\nabla}\right)$, increase sheath dissipation through the open field lines, etc. Measurements and analysis of the radial electric field and fluctuations with RMP application in tokamaks, helical devices and RFPs have been conducted in several devices, TEXTOR-DED [101], Tore Supra [102,103], TEXT [104,105], CSTN-III [106], MAST [107,108], ASDEX-Upgrade [109,110], DIII-D [111], LHD [112], and RFX-mod [113].

In TEXTOR-DED, it has been observed that the RMP application leads to the suppression of blob transport [114]. This is considered due to the suppression of large scale turbulence structure with RMP application by changing the mode structure from $k_{/ /} \approx 0$ (without an RMP) to finite $k_{/ /}$(with an RMP), as observed in experiments [102,101], which 
then reduces the blob size. This is also confirmed in numerical simulations with ATTEMPT [115]. Moreover, the enhanced sheath dissipation caused by the increased volume of open field lines with an RMP is responsible for the reduction of the blob radial velocity as observed in the numerical analysis in ref.[116].

In the experiments of TEXTOR-DED [117] and MAST [108], the reduction of the long range correlation of potential fluctuations during RMP application has been observed, which suggests the reduction of zonal flows. Possible mechanisms for this effect might be the suppression of large turbulent structures due to the nonzero $k_{/ /}$with RMP as mentioned above, and also the decrease in the Reynolds stress which is a drive for the zonal flows. However, observation in ASDEX-Upgrade showed that with a MP there is little change in the relative fluctuation level of the ion saturation current, $\delta I_{\text {sat }} / I_{\text {sat }}$, in the SOL [109]. The details of the mechanism are under investigation in refs.[118,119,120] with sophisticated models.

It is also noted that clear changes of the plasma potential profile as well as the edge electric field have been observed [101,107,105,109], where the radial electric field tends to change from negative (inward) to positive (outward). This is interpreted as due to the effect of open field lines produced by an RMP, where the fast escaping electrons compared to the ions must develop a positive field to restore the ambipolarity. In DIII-D [111], the radial profiles of plasma potential, electron temperature, poloidal electric field, DC ExB flux and turbulent radial particle transport inside the LCFS are all modified by a MP when the C-coil current $\left(\mathrm{I}_{\mathrm{c}}\right)$ increases from 1 to $3 \mathrm{kA}$.

On top of these, the very recent experiments in ASDEX-Upgrade [110] have reported a variety of plasma responses to the MP application, i.e. change of electric field and of turbulent transport differs depending on the plasma parameters, MP modes and amplitude etc.

These results suggest that the analysis of electric field formation and turbulent transport under MP application, including plasma response against MP penetration, is an urgent issue to be addressed for identification of the impacts of MP on the divertor functions and plasma-wall interaction.

\section{Summary}


The impacts of the 3D edge magnetic field geometry on the SOL and divertor transport have been discussed in regard to the divertor density regime, impurity screening and detachment control. The 3D effects are defined as it emerges when the $\perp$-transport starts to compete with //-transport in the open field lines of the stochastic layer or in the magnetic island structure for delivering plasma quantities (particle, energy, momentum) from the upstream region (around LCFS) to the divertor, i.e., $\Gamma_{/ /}\left(\frac{B_{r, \theta}}{B_{t}}\right)^{2} \sim \Gamma_{\perp}$. This condition is met in the stochastic layer or in the edge islands in the island divertor configurations, with typically $B_{r} / B_{t}=10^{-4} \sim 10^{-3}$, while it is not met in the $2 \mathrm{D}$ axi-symmetric configuration with an X-point divertor $B_{\theta} / B_{t} \sim 0.1$ and $\Gamma_{/ /}\left(\frac{B_{\theta}}{B_{t}}\right)^{2}>\Gamma_{\perp}$. For the momentum transport, the direction of field line connection to the divertor plates, i.e., plasma flow direction, becomes important. The spatial separation of opposite vector quantities, $\lambda_{m}$, the distance between the counter-streaming flows, is a key parameter to determine the $\perp$ loss of //-momentum through viscosity between the counter flows.

Based on this picture, the controlling parameters of the 3D effects have been discussed for the individual divertor functions. Table 1 summarizes the parameters together with the experimental observations, devices, and physical interpretations.

It is shown that for the divertor density regime the parameterization with the loss of //-momentum through viscosity between counter-flows, $\frac{\tau_{m / /}}{\tau_{m \perp}}$, and the replacement of //-energy flux with $\perp$-flux, $\frac{q_{\perp e}}{q_{/ / e}}$, appears to be a good measure for the presence or absence of the high recycling regime. It is noted that the interpretation applies to the attached phase only, while in a detached phase more complex physics come into play, such as atomic or molecular processes, ionization front shift to the upstream region etc. The absence of high recycling regime, which can occur in the domain indicated in Fig.5 might have an impact on the pumping efficiency due to the low divertor (neutral) density as well as on the physical sputtering due to the slow decrease of the divertor temperature. These effects have to be discussed further taking into account the divertor and baffle structure, which affect the neutral 
distribution. Also the available pumping speed, fuelling efficiency, operation density range are all influenced by 3D plasma distributions. The slow decrease of the divertor temperature against the upstream density increase can shift the detachment onset to higher densities. The higher $n_{u p}$ is preferred for impurity screening by suppressing the ion thermal force as discussed in Section 3. The value of $n_{u p}$ also can affect core plasma confinement.

With respect to the impurity screening or core decontamination with the 3D divertor configuration (stochastic edge or island divertor), it turned out that parameterization with the edge transport effects such as the friction force, $\frac{D_{s t}}{D_{\perp}}$, ion thermal force, $\frac{q_{\perp i}}{q_{/ / i}}$, does not work very well, although these effects have been discussed much in the literature. Yet there are still several issues to be assessed for the impurity transport, i.e. source location, injection energy, definition of screening (the ratio of impurity influx at the LCFS to the impurity source at the PFC), operation range for screening (density or collisionality dependence), effects of drift, electric field, turbulence and core transport, which are all missed in the present analysis.

The effects on the detachment stabilization have been clearly demonstrated in W7-AS, LHD and Tore Supra by changing the edge magnetic geometry. This indicates the possibility to control the $3 \mathrm{D}$ edge radiation structure with a magnetic field geometry. The key parameters are the distance between the divertor or edge island and core plasma, $\Delta x_{L C F S-i s l a n d}$, $\Delta x_{L C F S-d i v}$. It is found that that the larger $\Delta x_{L C F S-i s l a n d}, \Delta x_{L C F S-d i v}$ are, the better the detachment is stabilized, indicating that the decoupling of the core and edge in terms of particle fuelling is important.

In addition, there has been a variety of plasma behavior, i.e. change of electric field, turbulent transport and plasma response, against the MP application reported in various devices, as discussed in Section 5. The underlying mechanism is not yet clear. It is noted that, the RMP application does have clear effects on the transport and thus on the analysis presented in the preceded sections here through, for example, the values of $D_{\perp}, \chi_{\perp}$, used in the parameters, and ExB drift on the impurity transport etc. A comprehensive picture of the 3D effects on the SOL and divertor transport still must wait for these issues to be disclosed. 


\section{Acknowledgements}

The authors are grateful to Dr. A. Loarte and Dr. T. Takizuka for their valuable comments. 


\section{References}

1 N. Ohyabu et al., Nucl. Fusion 34 (1994) 387.

2 R. Koenig et al., Plasma Phys. Control. Fusion 44 (2002) 2365.

3 E. Strumberger, Nucl. Fusion 7 (1996) 891.

4 C. Alejaldre et al., Plasma Phys. Control. Fusion 41 (1999) A539.

5 D.T. Anderson et al., Fusion Sci. Technol. 50 (2006) 171.

6 T. Mizuuchi et al., J. Nucl. Mater. 176-177 (1990) 1070.

$7 \mathrm{Ph}$. Ghendrih, A. Grosman and H. Capes, Plasma Phys. Control. Fusion 38 (1996) 1653.

8 Ph. Ghendrih et al., Nucl. Fusion 42 (2002) 1221.

9 K.H. Finken et al., Nucl. Fusion 39 (1999) 637.

10 T.E. Evans et al., Nature Physics 2 (2006) 419.

11 O. Schmitz et al., J. Nucl. Mater. 438 (2013) S194.

12 T.E. Evans et al., Nucl. Fusion 53 (2013) 093029.

13 W. Suttrop et al., Phys. Rev. Lett. 106 (2011) 225004.

14 R. Fischer et al., Plasma Phys. Control. Fusion 54 (2012) 115008.

15 Y. Liang et al., Nucl. Fusion 50 (2010) 025013.

16 Y. Liang et al., Nucl. Fusion 53 (2013) 073036.

17 A. Loarte et al., Nucl. Fusion 54 (2014) 033007.

18 J-W. Ahn et al., Plasma Phys. Control. Fusion 56 (2014) 015005.

19 Y. Feng et al., Contrib. Plasma Phys. 54 (2014) 426.

20 D. Reiter et al., Fusion Sci. Technol. 47 (2005) 172, http://www.eirene.de/.

21 G. Ciraolo et al., Contrib. Plasma Phys. 54 (2014) 432.

22 P. Tamain et al., J. Computational Physics 229 (2010) 361.

23 C. Colin et al., PSI2014, O-24.

24 A.M. Runov et al., Phys. Plasmas 8 (2001) 916.

25 R. Zagorski et al., Nucl. Fusion 48 (2008) 024013.

26 H. Yamada et al., Fusion Sci. Technol. 58 (2010) 12.

27 O. Schmitz et al., Nucl. Fusion 48 (2008) 024009.

28 O. Schmitz et al., Plasma Phys. Control. Fusion 50 (2008) 124029.

29 M. Hirsch et al., Plasma Phys. Control. Fusion 50 (2008) 053001.

30 O. Schmitz et al., J. Nucl. Mater. 415 (2011) S886.

31 Y. Feng et al., Nucl. Fusion 49 (2009) 095002.

32 M. Kobayashi et al., Fusion Sci. Technol. 58 (2010) 220.

33 H. Frerichs et al., Nucl. Fusion 52 (2012) 054008.

34 T. Lunt et al, Nucl. Fusion 52 (2012) 054013.

35 T. Lunt et al, J. Nucl. Mater. 438 (2013) S254.

36 K. Ida et al., New Journal of Physics 15 (2013) 013061.

37 K. Ida et al., "Evidence of Stochastic Region near a Rational Surface in Core Plasmas of LHD”, 23rd IAEA FEC (2010), EXS/5-2, Daejeon, Korea.

38 F. Nguyen et al., Nucl. Fusion 37 (1997) 743.

39 Y. Feng et al., Plasma Phys. Control. Fusion 53 (2011) 024009.

40 M. Wischmeier et al., PSI2014, R-3.

41 Y. Shimomura et al., Nucl. Fusion 23 (1983) 869.

42 J. Neuhauser et al., Plasma Phys. Control. Fusion 31 (1989) 1551.

43 A. Loarte et al., Nucl. Fusion 38 (1998) 331.

44 B. Lipschultz et al., J. Nucl. Mater. 220-222 (1995) 50.

45 B. Lipschultz et al., Fusion Science and Technology 51 (2007) 369.

46 N. Asakura et al., Nucl. Fusion 35 (1995) 381.

47 M. Groth et al., J. Nucl. Mater. 415 (2011) S530. 
48 P. Grigull et al., Plasma Phys. Control. Fusion 43 (2001) A175.

49 Y. Feng et al., Plasma Phys. Control. Fusion 44 (2002) 611.

50 Y. Feng et al., Nucl. Fusion 48 (2008) 024012.

51 S. Masuzaki et al., J. Nucl. Mater. 313-316 (2003) 852.

52 M. Clever et al., Nucl. Fusion 52 (2012) 054005.

53 P.C. Stangeby, Nucl. Fusion 33 (1993) 1695.

54 B. LaBombard et al., J. Nucl. Mater. 241-243 (1997) 149.

55 B. LaBombard et al., Phys. Plasmas 2 (1995) 2242.

56 Y. Feng et al., Contrib. Plasma Phys. 44 (2004) 57.

57 N. Ezumi et al., J. Plasma Fusion Res. SERIES 8 (2009) 429.

58 M. Kobayashi et al., J. Plasma Fusion Res. 85 (2009) 393, in Japanese.

59 A. Bader et al., Nucl. Fusion 53 (2013) 113036.

60 Y. Feng et al., Nucl. Fusion 46 (2006) 807.

61 H. Frerichs et al., Nucl. Fusion 52 (2012) 023001.

62 M.Z. Tokar et al., Phys. Plasmas 11 (2004) 4610.

63 P. C. Stangeby, "The Plasma Boundary of Magnetic Fusion Devices”, Institute of Physics Publishing (2000).

64 A. Loarte Plasma Phys. Control. Fusion 43 (2001) R183.

65 A. Loarte J. Nucl. Mater. 241-243 (1997) 118.

66 D.P. Stotler et al., J. Nucl. Mater. 266-269 (1999) 947.

67 B. LaBombard et al., J. Nucl. Mater. 241-243 (1997) 149.

68 B. Meslin et al., J. Nucl. Mater. 266-269 (1999) 318.

69 M. Lehnen et al., J. Nucl. Mater. 337-339 (2005) 171.

70 H. Renner et al., Nucl. Fusion 40 (2000) 1083.

71 A. Loarte, Contrib. Plasma Phys. 32 (1992) 468.

72 C.J. Lasnier et al., Nucl. Fusion 38 (1998) 1225.

73 K. Hoshino et al., J. Nucl. Mater. 415 (2011) S549.

74 T. Eich et al., Nucl. Fusion 53 (2013) 093031.

75 J.R. Harrison et al., PSI2014 O-25.

76 S. Lippmann et al., Nucl. Fusion 31 (1991) 2131.

77 C. Breton et al., Nucl. Fusion 31 (1991) 1774.

78 G. Telesca et al., J. Nucl. Mater. 390-391 (2009) 227.

79 M. Kobayashi et al., Nucl. Fusion 53 (2013) 033011.

80 S. Morita et al., Nucl. Fusion 53 (2013) 093017.

81 R. Burhenn et al., Fusion Science \& Technology 53 (2004) 115.

82 I. Garcia-Cortes et al., Fusion Sci. Technol. 50 (2006) 307.

83 Y. Corre et al., Nucl. Fusion 47 (2007) 119.

84 M. Lehnen et al., Plasma Phys. Control. Fusion 47 (2005) B237.

85 R. Dei-cas and A. Samain, Plasma Phys. Control. Nucl. Fusion Res. 1 (1975) 563.

86 A.A. Shishkin, Nucl. Fusion 21 (1981) 603.

87 A. Samain, A. Grosman and W. Feneberg, J. Nucl. Mater.111-112 (1982) 408.

88 A. Samain et al, J. Nucl. Mater. 128-129 (1984) 395.

89 A. Nicolai, F. Schoengen and D. Reiter, Plasma Phys. 27 (1985) 1479.

90 M.Z. Tokar et al., Plasma Phys. Control. Fusion 39 (1997) 569.

91 M.B. Chowdhuri et al., Phys. Plasmas 16 (2009) 062502.

92 T.E. Evans et al., J. Nucl. Mater. 196-198 (1992) 421.

93 P. Grigull et al., J. Nucl. Mater. 313-316 (2003) 1287.

94 Y. Feng et al., Nucl. Fusion 45 (2005) 89.

95 M. Kobayashi et al., Nucl. Fusion 53 (2013) 093032. 
96 M. Kobayashi et al., Phys. Plasmas 17 (2010) 056111.

97 E.A. Drapiko et al., Nucl. Fusion 51 (2011) 073005.

98 B.J. Peterson et al., Plasma and Fusion Research 8 (2013) 2402037.

99 M. Kobayashi et al., "Effects of magnetic field structure of edge stochastic layer on radiative divertor operation in LHD”, Joint 19th ISHW and 16th IEA - RFP workshop 16 - 20 September 2013, Padova, Italy.

100 Y. Liang et al., Phys. Rev. Lett. 94 (2005) 105003.

101 Y. Xu et al., Nucl. Fusion 47 (2007) 1696.

102 J. Payan et al., Nucl. Fusion 35 (1995) 1357.

103 P. Devynck et al., Nucl. Fusion 42 (2002) 697.

104 S.C. McCool et al., Nucl. Fusion 29 (1989) 547.

105 A.J. Wootton, J. Nucl. Mater. 176-177 (1990) 77.

106 S. Takamura et al., Nucl. Fusion 28 (1988) 183.

107 P. Tamain et al., Plasma Phys. Control. Fusion 52 (2010) 075017.

108 J.R. Robinson et al., Plasma Phys. Control. Fusion 54 (2012) 105007.

109 H. W. Muller et al, J. Nucl. Mater. 438 (2013) S64-S71.

110 G.D.Conway et al., Plasma Phys. Control. Fusion 57 (2015) 014035.

111 J. A. Boedo et al., "Plasma Response to Applied Resonant Magnetic Perturbations in DIII-D”, 38th EPS Conference on Plasma Physics (2011), P1.122.

112 H. Tanaka et al., Phys. Plasmas 17 (2010) 102509.

113 P. Scarin et al., 23rd IAEA FEC (2010), Daejeon, Korea.

114 Y. Xu et al., Nucl. Fusion 49 (2009) 035005.

115 D. Reiser, Phys. Plasmas 14 (2007) 082314.

116 O.E. Garcia et al., Phys. Plasmas 13 (2006) 082309.

117 Y. Xu et al., Nucl. Fusion 51 (2011) 063020.

118 P. Beyer et al., Plasma Phys. Control. Fusion 44 (2002) 2167.

119 M. Leconte and P.H. Diamond, Phys. Plasmas 18 (2011) 082309.

120 M. Leconte, P.H. Diamond and Y. Xu, Nucl. Fusion 54 (2014) 013004. 


\section{Table 1}

Table 1. Summary of the 3D effects on the SOL and divertor transport in various devices. $†$ : Numerical simulations. The definitions of formulae are given in the text.

\begin{tabular}{|c|c|c|c|c|}
\hline Observations & Devices & Key parameters & Interpretation & Divertor functions \\
\hline \multirow{2}{*}{$\begin{array}{c}n_{d i v} \propto n_{L C F S}{ }^{\alpha+1} \\
T_{d i v} \propto n_{L C F S}{ }^{\alpha} \\
\alpha=2 \rightarrow \leq \mathbf{1} \\
\text { (weak div-LCFS coupling) }\end{array}$} & \multirow{2}{*}{$\begin{array}{l}\text { W7-AS, LHD, } \\
\text { TEXTOR-DED, } \\
\text { HSX' }^{+}\end{array}$} & $\tau_{m / /} / \tau_{m \perp} \gg 1$ & $\begin{array}{l}\quad / / \text {-momentum loss } \\
\rightarrow p_{\text {LCFS }}>p_{d i v}\end{array}$ & \multirow{2}{*}{$\begin{array}{c}\text { Pumping efficiency } \downarrow \\
\text { Phys. Sputtering } \uparrow \\
\text { Detach. onset density } \uparrow \text { (?) }\end{array}$} \\
\hline & & $\begin{array}{l}q_{\perp e} / q_{/ / e} \gg 1 \\
q_{/ / \text {conv }} / q_{/ / \text {cond }}\end{array}$ & $\begin{array}{l}\text { Reduction of } / / \text {-energy } \\
\text { conduction }\end{array}$ & \\
\hline \multirow{3}{*}{ Core decontamination } & \multirow{3}{*}{$\begin{array}{l}\text { TEXT, Tore Supra, } \\
\text { W7-AS, W7-X†, LHD, } \\
\text { TEXTOR-DED, TJ-II }\end{array}$} & $D_{s t} / D_{\perp} \gg>1$ & Enhanced friction force & \multirow{3}{*}{ Impurity screening $\uparrow$} \\
\hline & & $q_{\perp i} / q_{/ / i} \gg>1$ & Ion thermal force suppression & \\
\hline & & $\lambda_{s t-S O L} / \lambda_{i m p} \lambda$ & $\begin{array}{l}\text { Shallow penetration of neutral } \\
\text { impurity }\end{array}$ & \\
\hline \multirow[b]{2}{*}{ Detach. stabilization } & \multirow{2}{*}{$\begin{array}{l}\text { W7-AS, } \\
\text { LHD, } \\
\text { Tore Supra }\end{array}$} & $w_{\text {island }}$ & Radiation modulation by islands & \multirow[b]{2}{*}{ Heat removal $\uparrow$} \\
\hline & & $\begin{array}{c}\Delta x_{L C F S-d i v} \\
\Delta x_{L C F S-i s l a n d}\end{array}$ & $\begin{array}{l}\text { Core-edge decoupling } \\
\rightarrow \text { particle fueling } \downarrow \\
\text { core impurity penetration } \downarrow\end{array}$ & \\
\hline MARFE onset delayed & TEXTOR-DED & $f_{R M P}$ & $\begin{array}{l}\text { Avoid localized cooling by } \\
\text { spreading recycling region with } \\
\text { RMP rotation }\end{array}$ & Density limit $\uparrow(?)$ \\
\hline
\end{tabular}




\section{Figure captions}

Fig. 1 Schematics of transport in stochastic field lines (top) and in magnetic island (bottom). $\Gamma_{/ /, \perp}$ represents the flux of any physical quantity (particle, momentum, energy) that are parallel and perpendicular to the magnetic field lines, respectively.

Fig. 2 Schematics of formation of //-flow towards divertor plates in 2D axi-symmetric (top) and in 3D divertor (bottom) configurations. In the 2D case, the flux tubes are connected to inner and outer divertor plates in toroidally opposite directions $(+/-\phi)$ each other. The similar situation takes places at the flux tubes in the $3 \mathrm{D}$ case, where the magnetic island structure is opened and connected to the divertor plates. In the 3D case, the spatial separation between counter-streaming flows, $\lambda_{m}$, becomes short and thus momentum loss of //-flow via $\perp$-viscosity takes place.

Fig. 3 Schematics of deformation of flux tubes in the stochastic layers. Through bending of flux tubes by $B_{r}$ and stretching by magnetic shear, the flux tubes of long and short $\mathrm{L}_{\mathrm{C}}$ are "squeezed." The resultant interaction area increases and $\perp$ exchange of plasma quantity is enhanced with substantial plasma density causing viscosity.

Fig. 4 Dependence of (a) $n_{\text {down }}$ (b) $T_{\text {down }}$ on upstream density $n_{u p}$, including $\perp$ loss of //-momentum, obtained by extended two point model [60]. With 
increasing the momentum loss factor, $f_{m 0}$, the upstream and downstream coupling becomes weak and the density dependence deviates from the high-recycling regime in a $2 \mathrm{D}$ axi-symmetric divertor.

Fig. 5 Divertor density regime of various devices in terms of $\perp$ loss of //-momentum, $\tau_{m / /} / \tau_{m \perp}$, and replacement of //-energy flux with $\perp$-flux, $q_{\perp e} / q_{/ / e}$. Red: no high recycling regime $(\alpha<3)$, blue: with high recycling regime $(\alpha<3)$, where $n_{\text {down }} \propto n_{u p}{ }^{\alpha}$. The results from numerical simulations are plotted with dashed lines. The larger the $\tau_{m / /} / \tau_{m \perp}$ or $q_{\perp e} / q_{/ / e}$ are, the weaker the upstream and downstream coupling, i.e., density dependence, is.

Fig. 6 Operation domain of various devices in the space of $\frac{\lambda_{s t-S O L}}{\lambda_{i m p}}$ and $\frac{D_{s t}}{D_{\perp}}$, where the observation of impurity screening is indicated with blue circles, and the observation of no impurity screening with red circles. The results from numerical simulations are plotted with dashed lines. $\frac{\lambda_{s t-S O L}}{\lambda_{i m p}}$ is the ratio of the radial width of the stochastic layer or island divertor SOL to neutral impurity penetration length for carbon. $\frac{D_{s t}}{D_{\perp}}$ represents the radially outward particle flux enhancement.

Fig. 7 Operation domain for stable detachment in W7-AS, which is indicated with 
the shaded region, in terms of the distance between the divertor plate and the LCFS, $\Delta x_{L C F S-d i v}$, and $L_{C}[94,93]$. Larger $\Delta x_{L C F S-d i v}$ and shorter $L_{C}$ are preferred for stable detachment.

Fig. 8 Operation domain for stable detachment in LHD in terms of the distance between the island X-point and the LCFS, $\Delta x_{L C F S-i s l a n d}$, and the RMP strength, $B_{r} / B_{t}$ [95]. Stable detachment : circles. Radiation collapse : triangles. Larger $\Delta x_{L C F S-i s l a n d}$ and larger $B_{r} / B_{t}$ are preferred for stable detachment. 
Fig. 1 Single-column $(68 \mathrm{~mm})$

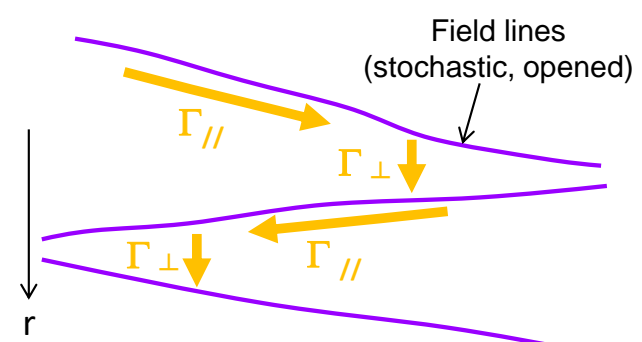

Divertor plates

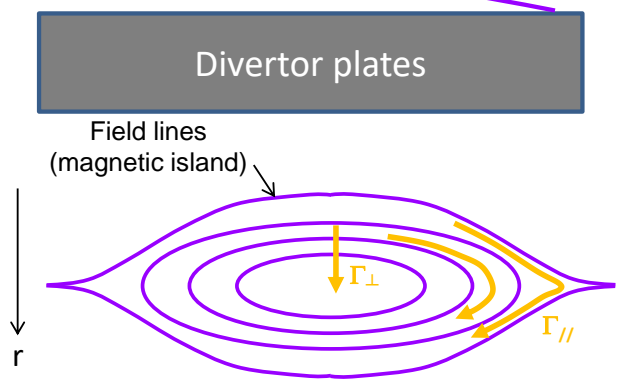

Fig.1 Schematics of transport in stochastic field lines (top) and in magnetic island

(bottom). $\Gamma_{/ /, \perp}$ represents flux of any physical quantity (particle, momentum, energy)

that are parallel and perpendicular to the magnetic field lines, respectively. 
Fig. 2 Single-column $(90 \mathrm{~mm})$

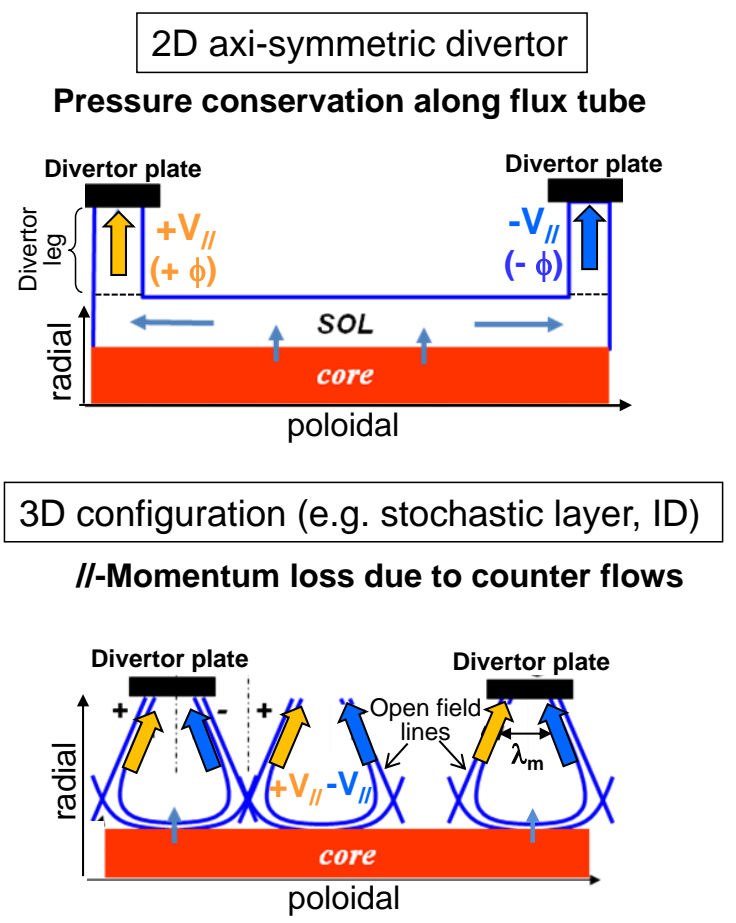

Fig.2 Schematics of formation of //-flow towards divertor plates in 2D axi-symmetric (top) and in 3D divertor (bottom) configurations. In the 2D case, the flux tubes are connected to inner and outer divertor plates in toroidally opposite directions $(+/-\phi)$ each other. The similar situation takes places at the flux tubes in the 3D case, where the magnetic island structure is opened and connected to the divertor plates. In the 3D case, the spatial separation between counter-streaming flows, $\lambda_{m}$, becomes short and thus momentum loss of //-flow via $\perp$-viscosity takes place. 
Fig. 3 Single-column $(70 \mathrm{~mm})$

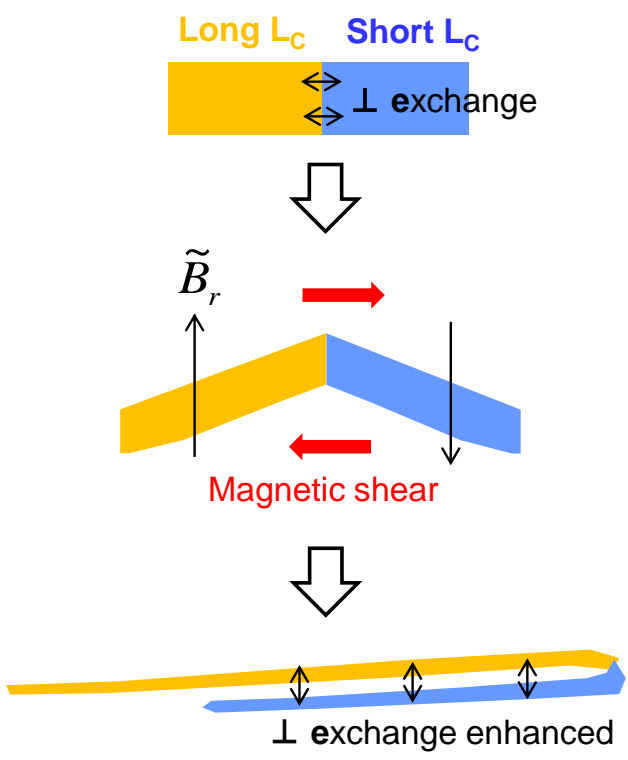

Fig.3 Schematics of deformation of flux tubes in the stochastic layers. Through bending of flux tubes by $B_{r}$ and stretching by magnetic shear, the flux tubes of long and short $\mathrm{L}_{\mathrm{C}}$ are "squeezed." The resultant interaction area increases and $\perp$ exchange of plasma quantity is enhanced with substantial plasma density causing viscosity. 
Fig. 4 Single-column $(90 \mathrm{~mm})$
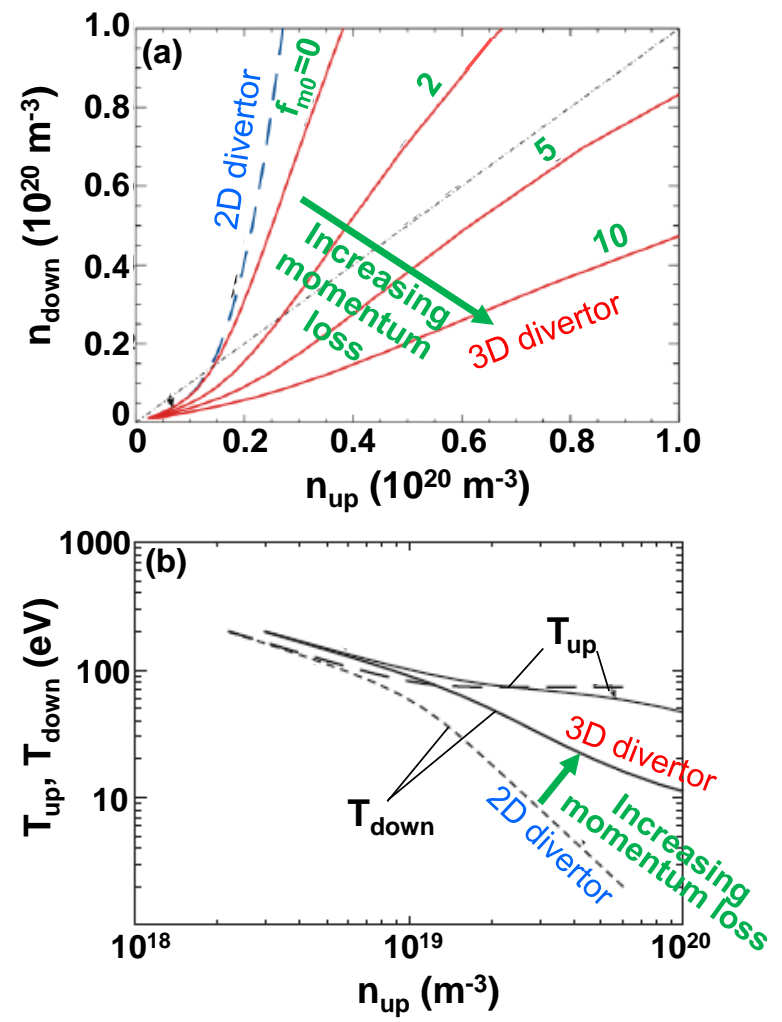

Fig.4 Dependence of (a) $n_{\text {down }}$ (b) $T_{\text {down }}$ on upstream density $n_{u p}$, including $\perp$ loss of //-momentum, obtained by extended two point model [60]. With increasing the momentum loss factor, $f_{m 0}$, the upstream and downstream coupling becomes weak and the density dependence deviates from the high-recycling regime in a 2D axi-symmetric divertor. 
Fig. 5 Single-column $(68 \mathrm{~mm})$

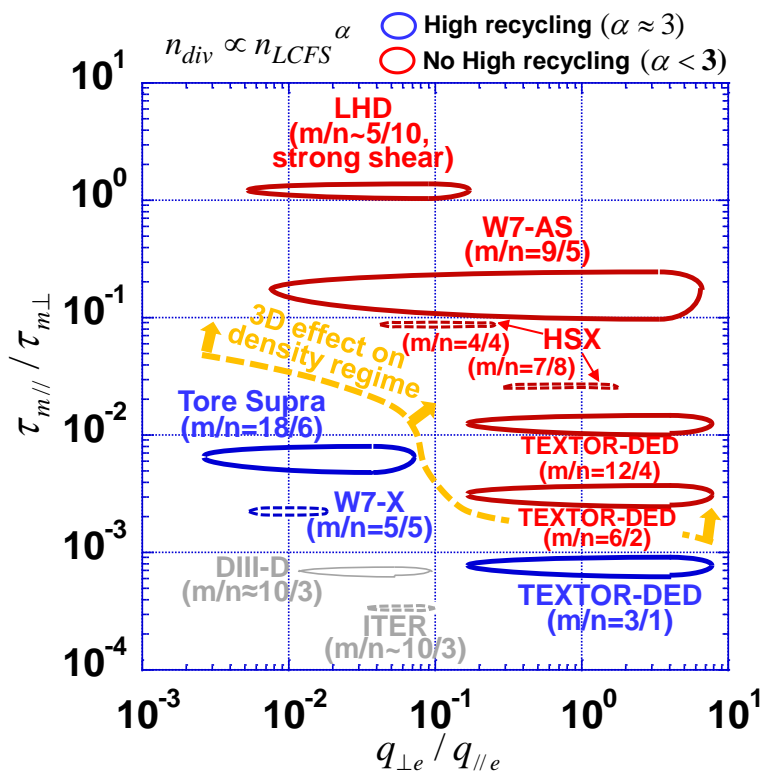

Fig.5 Divertor density regime of various devices in terms of $\perp$ loss of //-momentum, $\tau_{m / /} / \tau_{m \perp}$, and replacement of //-energy flux with $\perp$-flux, $q_{\perp e} / q_{/ / e}$. Red: no high recycling regime $(\alpha<3)$, blue: with high recycling regime $(\alpha<3)$, where $n_{\text {down }} \propto n_{u p}{ }^{\alpha}$. The results from numerical simulations are plotted with dashed lines. The larger the $\tau_{m / /} / \tau_{m \perp}$ or $q_{\perp e} / q_{/ / e}$ are, the weaker the upstream and downstream coupling, i.e., density dependence, is. 
Fig. 6 Single-column $(68 \mathrm{~mm})$

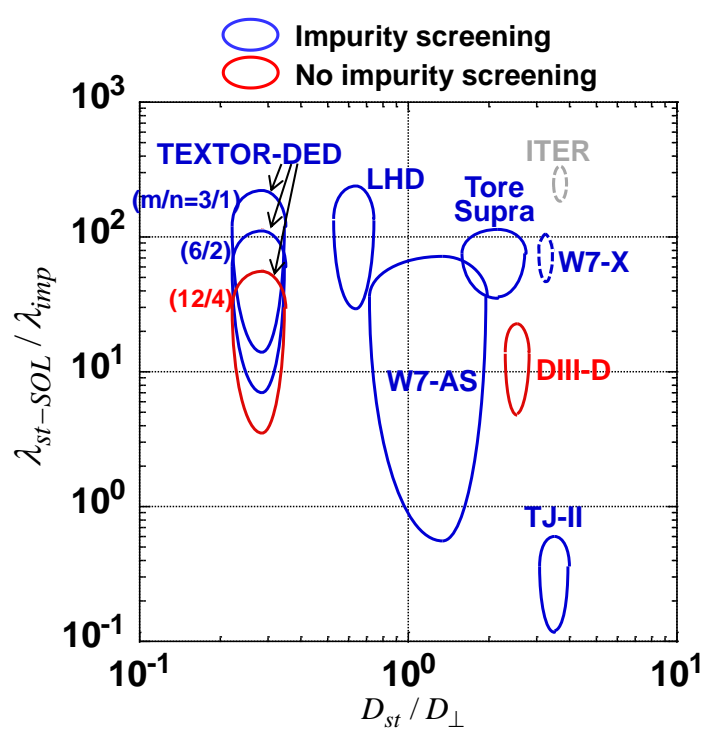

Fig.6 Operation domain of various devices in the space of $\frac{\lambda_{s t-S O L}}{\lambda_{i m p}}$ and $\frac{D_{s t}}{D_{\perp}}$, where the observation of impurity screening is indicated with blue circles, while no impurity screening with red circles. The results from numerical simulations are plotted with dashed lines. $\frac{\lambda_{s t-S O L}}{\lambda_{\text {imp }}}$ is the ratio of the radial width of the stochastic layer or island divertor SOL to neutral impurity penetration length for carbon. $\frac{D_{s t}}{D_{\perp}}$ represents the radially outward particle flux enhancement. 
Fig. 7 Single-column $(80 \mathrm{~mm})$

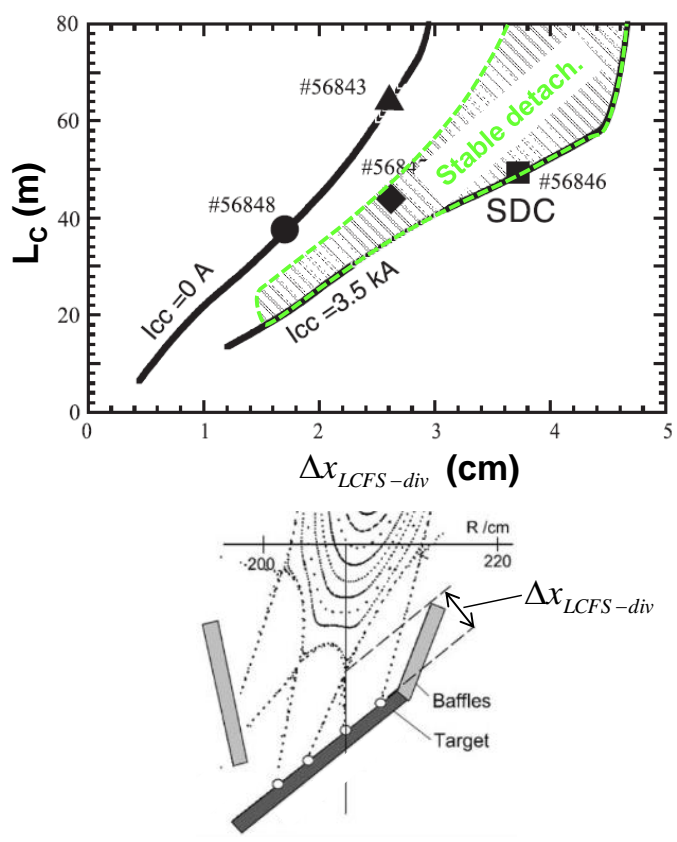

Fig.7 Operation domain for stable detachment in W7-AS, which is indicated with the shaded region, in terms of the distance between the divertor plate and the LCFS, $\Delta x_{L C F S-d i v}$, and $L_{C}[94,93]$. Larger $\Delta x_{L C F S-d i v}$ and shorter $L_{C}$ are preferred for stable detachment. 
Fig. 8 Single-column $(80 \mathrm{~mm})$

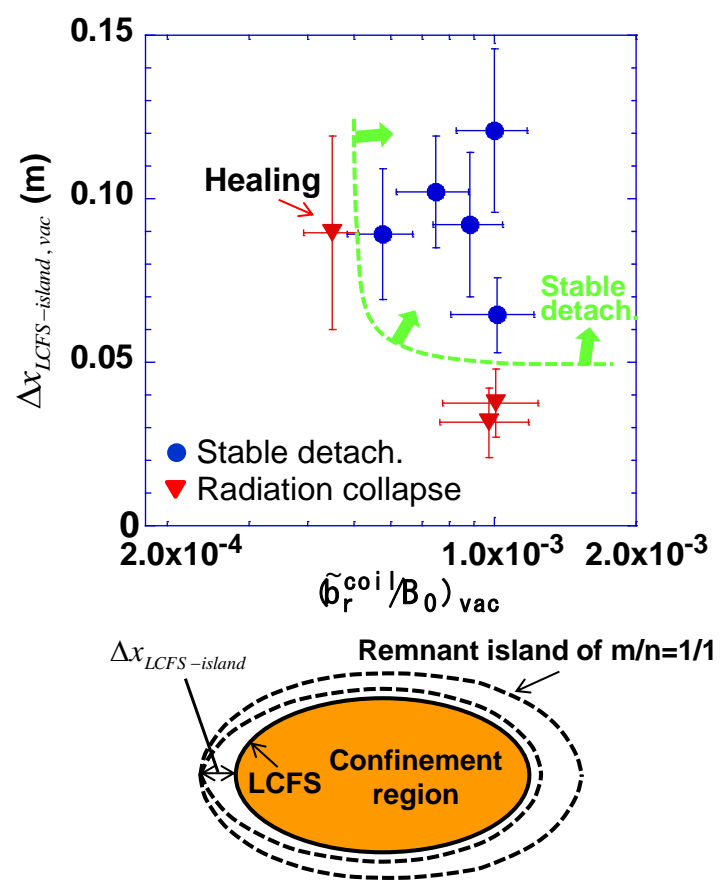

Fig.8 Operation domain for stable detachment in LHD in terms of the distance between the island X-point and the LCFS, $\Delta x_{L C F S-i s l a n d}$, and the RMP strength, $B_{r} / B_{t}$ [95]. Stable detachment : circles. Radiation collapse : triangles. Larger $\Delta x_{L C F S-i s l a n d}$ and larger $B_{r} / B_{t}$ are preferred for stable detachment. 